\section{One-Line Proof of the AM-GM Inequality}

\author{
O. A. S. Karamzadeh
}

$\longrightarrow$ he AM-GM inequality says $\frac{x_{1}+x_{2}+\cdots+x_{n}}{n} \geq \sqrt[n]{x_{1} x_{2} \cdots x_{n}}$ (all $x_{i}>0$ ). This is perhaps the best known and most useful nontrivial inequality in mathematics, with a large number of interesting proofs in the literature. A very clever proof of this inequality, which appeared of late in [1], has given me motivation to communicate a very short proof of it, which I used to present to Iran's Olympiad team, preparing for the IMO, several years ago.

Let me, before going any further, give a generalization of the AM-GM inequality in the case $n=2$. It is trivial to see that, whenever $0<a \leq b$ and $x>0, a \leq x \leq b$ if and only if $a+b \geq x+\frac{a b}{x}$. The latter inequality is stronger than the AMGM inequality for $n=2$ (indeed, just choose $x=\sqrt{a b}$ ). It also allows equality only for either $x=a$ or $x=b$.

Now the quick proof of the general case goes as follows. If $x_{1}=x_{2}=\cdots=x_{n}$, then we are done. If not, put $x_{1} x_{2} \cdots x_{n}=g^{n}$, and without loss we may assume $x_{1} \leq x_{i}$ and $x_{2} \geq x_{i}$ for all $i$, hence $x_{1}<g<x_{2}$. (We have noted that $x_{1}<g<x_{2}$ if and only if $x_{1}+x_{2}>g+\frac{x_{1} x_{2}}{g}$.) Therefore $x_{1}+x_{2}+\cdots+x_{n}>g+\frac{x_{1} x_{2}}{g}+x_{3}+\cdots+x_{n}$; but the latter expression is $\geq g+(n-1)^{g} g=n g$, by the inductive hypothesis (noting that $\frac{x_{1} x_{2}}{g} x_{3} \cdots x_{n}=g^{n-1}$ ).

REMARK The last part of the above proof shows that if not all $x_{i}^{\prime}$ 's are equal, then in fact we have $\frac{x_{1}+x_{2}+\cdots+x_{n}}{n}>$ $\sqrt[n]{x_{1} x_{2} \cdots x_{n}}$, that is to say, the equality $\frac{x_{1}+x_{2}+\cdots+x_{n}}{n}=$ $\sqrt[n]{x_{1} x_{2} \cdots x_{n}}$ holds only when $x_{1}=x_{2}=\cdots=x_{n}$. The latter fact does not follow automatically from most of other proofs of this inequality in the literature.

\section{REFERENCE}

[1] Michael D. Hirschhorn, "The AM-GM Inequality," Mathematical Intelligencer 29 (2007), nos. 4, 7.

Department of Mathematics

Chamran University

Ahvaz

Iran

e-mail: karamzadeh@ipm.ir 